\title{
THE ASSOCIATION OF OCULAR AND NASAL ACCESSORY SINUS DISEASE.*
}

\author{
By G. Oram Ring, A. M., M. D., \\ Phil,Adel,phia.
}

It will be conceded readily that the careful study of the ocular symptomatology, either associated with or directly caused by disease of the sinuses accessory to the nose, constitutes the focal point to which converge two important specialties.

To the ophthalmologist the early recognition of this association is always an important matter, and his failure may be an exceedingly grave one.

It has long been recognized that the etiology of the often complex symptomatology of this anatomic and clinical borderland may require for its elucidation the cooperation of the internist, the laboratory and X-ray experts, in association with the rhinologist and ophthalmic surgeon.

Some of the ophthalmologists present will recall the discussion precipitated some ten years ago by. Dr. Posey in a paper before the American Ophthalmological Society upon the position of the ophthalmologist in the treatment of nasal sinus disease, the plea being made for a more accurate appreciation and study of the relationship obtaining between these ocular and nasal structures.

In his discussion of Posey's paper, the late Dr. Gruening of distinguished, if somewhat bellicose memory, replied, "The ophthalmologists do pay attention to these conditions, and the ophthalmologist must be a rhinologist; that is understood, and there can be no doubt about it."

The enormous development of each of our specialties during the last ten years may well make us question this dictum of Teutonic finality.

\footnotetext{
- Read by Invitation in a symposium before the Section of Otology and Rhinology, College of Phyzlclans, Phlladelphla, Pa.
} 
It was Arthur Christopher Benson who said of the specialist that he was harmless and necessary, so long as he was aware of his limitations.

The ophthalmologist who has the temerity to attempt an entrance into the field that you so preeminently command, if he has had sufficient training to make him conversant with these associated conditions, may intelligently examine and conservatively treat them, but must be prepared to ask the assistance, at the proper time, of his rhinologic confreres in the performance of the necessary operations upon the contiguous sinuses.

I.et us try to disassociate our study from the stigma of undue magnification of the cause of the ocular symptomatology as necessarily resident in the sinuses, even though disease of both structures exists.

In the development of this thought it behooves us, likewise, to be constantly on guard to recognize the constitutional factors that may be inducing a toxemia of each of these contiguous structures at one and the same time, the proper treatment and removal of which may cause prompt amelioration and cure of the ocular and nasal conditions, without the need of other than the mildest local treatment.

The narrow viewpoint of the specialist was recently illustrated while discussing, with a distinguished professor of operative dentistry, the association of maxillary sinuitis with diseased teeth conditions, he stoutly maintaining that every such case was due to the teeth, and I think accepted, with considerable qualification, the writer's definite statement that about seventy-five per cent of such conditions were entirely disassociated with any dental disease.

Since the subject of accessory sinus disease, from the rhinologic standpoint, was so admirably reviewed in a recent symposium before the members of this section, participated in by such authorities as Skillern, Grayson, Van Sant, Coates, Gleason, Wood, Eves and others, it is my purpose to approach it in the main from the ocular viewpoint.

The rhinologist will unquestionably maintain that many cases of sinuitis are entirely minus any ocular signs, and indeed most of us will agree with so careful an observer as Jessup of I.ondon, when he suggests that, at times, there are 
no characteristic visual changes associating these two contiguous structures.

Bryan of Washington believes that the majority of all the severe inflammations of the accessory sinuses are accompanied with more or less disturbance of the eyes, the variations being, as a rule, governed by the severity of the sinus disease, and that they are not discovered early because not adequately sought until ocular symptoms develop.

T. E. Brawley of Chicago (Journal American Medical Association, March 23, 1907, "The Relation of Diseases of Accessory Nasal Sinuses to Diseases of the Eye"), a painstaking observer and student of these associated conditions. maintained, as early as 1907, that every case of intraocular disease should be accompanied by a careful study of these contiguous cavities. Even a partial review of the literature will make most of us willing to assume the advanced position recorded by the late lamented Reber, that all manner of extraand intraocular disease is, at times, traceable to nonsuppurative and suppurative pathologic processes in these contiguous nasal sinuses, and will certainly justify the observation that in all ocular diseases of obscure origin, the sinuses should be studied, and if ocular signs justify it, treatment of the suspected sinuses is indicated, even in spite of negative rhinologic report.

It is a fact that the careful special testing of visual function and the finding of certain changes in the visual field may establish a diagnosis before nasal symptoms have been sufficiently marked to attract attention, and further, it is not infrequent for closed empyemas and mucoceles to give a false sense of security by an almost total absence of nasal symptoms, notwithstanding the presence of ocular change.

It is quite impossible, in a comparatively brief consideration of the subject, to refer adequately to a very extensive literature which has accumulated during the last ten years, much of which may be classed as intensive and epoch making.

Onodi, as is perfectly well. known, has given special impetus to the whole subject we are considering by his contributions, which include a study of the oculoorbital, intracranial and cerebral complications as well as by his published anatomic 
studies of the relations of the posterior sinuses to the normal and anomalous proximity to the optic nerve.

The anatomic studies of $\mathrm{H}$. W. Loeb (AnNals of OtoLoGy, RHINOLOGY AND LARYNGOLOGY, June, 1909) likewise furnish illuminating data regarding the relation of the posterior sinuses to the nerve and chiasm, this distinguished writer having advised us that more than half of the nerve was included in the sinus portion.

The important relationship between the orbits and the sinuses, in the anterior segment of the skull, was the subject of an important communication by Dr. Samuel D. Risley, before the International Congress at Edinburgh in 1894, concerning abnormalities of ocular balance; it being even then recognized that departures from the normal contour in sinus development furnished a strong predisposing cause of interference with sinus drainage.

The strategic position occupied by these nasal sinuses with reference to orbital attack, by way of the roof, floor and inner wall, through the actual giving way of these thin, bony and mucoperiosteal partitions (and, indeed, in the case of the ethmoids, the existence of socalled dehiscences mark the absence of any but the latter protection), makes the direct extension by pressure, erosion and necrosis, or through the interstices, readily understood. Adhesions may occur between the orbital periosteum and the optic nerve sheath, and these adhesions may be vascularized, offering a direct route for ocular infection (Brawley).

It is quite unnecessary in this presence to trace the ophthalmic artery in its ramification from the orbit into the ethmoid and frontal cells, nor the return journey of the ethmoidal veins into their ophthalmic reservoir.

Again, the intimate association of the ciliary system need scarcely be urged as pointing to the ease with which a toxemia may reach the eyes. With the role played by the fifth cranial nerve in ophthalmic, sinus and intracranial disease you are also entirely conversant.

The roble of the lymphatics and the lymphatic sheaths of the nerves should be given emphasis in tracing the likely routes of infection. 
Regarding the latter method of transfer, it is claimed by Miodowski, as the result of his work. in the clinic of Professor Brieger of Breslau (quoted by Freudenthal), that, by reason of their anatomic features, they should be and. he believes are, the best reservoirs of all invading bacteria.

It would be very desirable, if it were possible, to separate the ocular symptoms induced by sinus disease into two definite groupings, the anterior and posterior.

The anatomic association, already referred to, as well as the numerous clinical reports at our service, will serve to indicate the impossibiltiy of any such dogmatic differentiation.

The routes of ocular involvement, secondary to rhinogenous disease, will be then-

1. By continuity.

2. By way of the blood vessels.

3 . By way of the lymphatics.

4. By way of the lymphatic sheaths of the nerves.

Ocular symptoms, the result of abnormalities in the nasal mucosa, have long been an established fact, entirely apart from the presence of pus producing organisms. Differences in the power of accommodation in the two eyes have frequently been relieved, as recorded by such authorities as Ballenger, Stucky, Pynchon and others, by the removal of nasal pressure in a partial excision of a hypertrophied middle turbinal. The disappearance of blepharospasm by the same procedure is a likewise generally recognized observation. I acrimal disease from epiphora to blenorrhea may easily be associated with mechanical obstruction of the nasal end of the duct. Indeed the vicious role played by the duct, in the transfer of infection to the cornea, is a generally conceded possibility, S. Lewis Ziegler, for example, claiming that upwards of 90 per cent of corneal ulcers are due to nasal conditions.

Blepharospasm, dacryocystitis, photophobia, epiphora. ocular pain and reflex ocular disturbance, expressing itself in socalled asthenopia, have many times found a causative factor in nasal congestion, spurs, tumors, ulceration, hypertrophied or polypoid turbinal degeneration and from contraction from nasal synechia caused by the too vigorous use of the cautery. W. C. Parker, in a review of these associated conditions, believes 50 per cent of them are caused by nasal disease. 
Ziem and Kuhnt have traced iritis and cyclitis to a nasal origin. Parker has found improvement in refractive defects to follow the lessening of ciliary irritation and congestion by nasal treatment, and few oculists today attempt to treat phlyctenular disease of the conjunctiva or cornea without taking into account the condition of the nose, the tonsils and the adenoids.

Stauffer of Salt J ake City has noted deep ciliary injection to be present in nearly all high deflections with pressure on the middle turbinal.

$\Lambda$ number of writers, among them Griffin and Haskell, have recorded observations pointing to the vascular or reflex connection between nasal hypertrophies and glaucoma, the latter disease being greatly relieved by the removal of the thickened vascular structure.

Middle turbinal pressure may be either against the septum or antral wall, and a number of well known authorities insist that if pressure prevents free ventilation and drainage, the turbinal should be removed, even though it presents no pathologic changes, as the negative pressure thus induced within the ethmoid or frontal will eventually lead to pain, pus formation and ocular disturbance.

If the conditions briefly outlined are productive of evident ocular symptoms, it is surely. not a far cry to the more profound lesions that may easily accompany the deeper sinus congestions, mucoceles or empyemas.

The sinus involvement may run the gamut from a simple hyperemia to catarrhal inflammation, suppuration and necrosis, and each form may, and frequently is, accompanied by ocular symptoms.

Perhaps the simplest form of ocular discomfort which is apt to succeed a congestion of the nasal mucosa, with obstruction of the normal sinus outlets, as pointed out by Parsons (Journal-Lancet, 1913, page 332), is a unilateral headache with pain and some tenderness at the upper, inner orbital angle, aggravated by stooping, with or without some vertigenous sensation, congestion of the conjunctiva of the lid and eyeball, with lacrimation, muscular twitching and discomfort upon attempting to use the eyes for near work. The latter symptom will probably draw attention to a refractive defect, which 
in itself may be an entirely minor element in the causation of the condition.

The vicious circle thus set into action, with the induction of socalled negative pressure within the sinuses, is a common observation. Middle turbinal pressure against the septum may be the contributive cause, and here recurrences may make it necessary to excise it in part.

As a rule the acute cases of sinus disease are not seen by the ophthalmic surgeon, as the family physician and nasal specialist are those consulted during the earlier manifestations, and unless the infection is an especially virulent one, causing external violent ocular symptoms, the opportunity to examine such expressions of disease is only afforded well on in the subacute or chronic stages.

The usual types of eye involvement in acute and chronic sinus disease are those with external manifestations such as edema, cellulitis, abscess; those without external signs but manifesting definite lesions of the ocular fundus; those with corneal, iritic, ciliary or general uveal inflammation and even glaucoma; those exhibiting the pressure signs of mucoceles; and finally the unilateral pain and headache due to rarefaction of air in frontal or ethmoid which often expresses itself as violent asthenopia with or without definite pupillary, paretic or paralytic muscle phenomena.

\section{SYMPTOMS.}

The ocular symptoms of sinus discase, to which I will first direct your attention, is edema of the eyelids, on one or both sides, usually unilateral. Gerber claims it to be a very early symptom of orbital involvement. It may be present for prolonged periods, at times being typically fugitive and recurrent, varying from a slight flushing to a marked swelling of red or brawny type. Dr. de Schweinitz in 1910 (American Ophthalmological Society) emphasized these fugacious appearances as pointing just as definitely to sinus disease as the common edema more generally present. They are usually accompanied with unilateral pain, supraorbital neuralgia and frequently with burning on the affected side of the face, and can be an expression of discase in any one of the four sinuses, but are thought to be conspicuously evident in acute ethmoiditis and 
frontal sinuitis. Edema tends to lessen as drainage is established, and if due to either of the latter sinuses is apt to be less in evidence at night and more in the morning. There is no doubt, however, that even a casual review of the literature will definitely place the maxillary sinus in the role of causation. Onodi, Digiuseppie, Di 'Tito, Rollet, Genet, Freeman of this city and others have reported cases. In Freeman's case it was caused by only a few drops of pus in the sinus. If due to disease of the maxillary antrum the edema should be more marked during the day, if the patient is in an upright position.

This symptom must be distinguished from that caused by incipient hordeolum, as well as from the edema of an acute dacryocystitis, that from lid furuncle, or from the deep infiltration that precedes an abscess of the lids.

The edema accompanying periostitis of the orbital margin can, as a rule, be diagnosticated by the hard character of the underlying swelling.

In erysipelas the swelling and redness are uniform, the skin feels thicker and harder, and circumscribed infiltration is absent. The swelling occupies both, as a rule, and usually extends to the neighboring parts. The recurring angeioneurotic edemas allied to urticaria, and often associated with disturbances of the menstrual period, must be considered as well as those symptomatic of migraine. The edema accompanying acute eczema is also worthy of note.

Consideration of the noninflammatory lid edemas, associated with cardiac disease and nephritis, often appearing under the guise of edema fugax, must also be differentiated.

The edema of acute blenorrhea and diphtheria of the conjunctiva, iridocyclitis, uveitis and glaucoma are, as a rule, rendily eliminated. The edema of a retrobulbar phlegmon or that expressive of a severe orbital cellulitis or abscess secondary to frontal or ethmoidal empyema must be, if possible, differentiated from the early orbital manifestation of thrombosis of the cavernous sinus. In thrombosis the lid and conjunctival swelling are marked, the eyeball is protruded and moves with difficulty. The retinal veins are enormously dilated; and there is apt to be a doughy edema in the mastoid region. In sinus thrombosis the edema and swelling are frequently bilateral, and this is rare in sinus disease. Thrombosis is apt very 
promptly to lead to cerebral symptoms and a fatal issue. (On the other hand, suppuration in the posterior ethmoid cells and maxillary antrum mav, according to Fuchs, lead to sinus thrombosis, hence it will be readily understood how difficult at times absolutely accurate differential diagnosis becomes.

Our cdema study can easily become more complex by the development of a meningitis, in association with oculoorbital disease of rhinogenous origin, and in this connection has been exhaustively studied by $\mathrm{P}$. H. Gerber (Komplikationen der Stirnhöhlentzundungen, 1909), with an analysis of fifty-one cases from literature, as well as by Onodi, in his well known work (Pathologie and Therapie der Nasenkrankheiten, 1910).

To these have recently been added two cases by Dr. Samuel Leopold of our city, one with oculoorbital symptoms and a second with rapid brain involvement from influenza without demonstrable ocular signs, the first illustrating an intermittent frontal sinuitis with a final meningitic attack, and the second quickly terminating in a sudden apoplectiform attack. A third slow, insidious, protracted form is distinguished. When ocular complications are present we may have disease of the inferior sinus wall or orbital roof. Thrombophlebitis may indirectly produce leptomeningitis. Here again Leopold believes the lymph channels may also play an important rôle.

In both tenonitis and orbital cellulitis, secondary to sinus disease, edema of the lid and conjunctiva and proptosis of the eyeball are present. Again, according to Fuchs, if the chemosis is pronounced and the proptosis slight, tenonitis is probably in evidence. 'Ihe opposite condition of affairs points to the deeper involvement.

Dr. Posey refers to a collateral inflammatory edema of the upper lids and orbit accompanying a periostitis in the presence of acute frontal and ethmoidal sinuitis, in which an exploratory puncture showed the presence of pus, under the periosteum, but to which an incision gave no added purulent matter.

Actual blackening of the lids at times occurs, suggestive of an ecchymosis, and this as the edema may be also recurrent. The value of this symptom was emphasized by de Schweinitz in his paper, "Some Observations of the Ocular Manifestations of Sinus Disease," before the American Ophthalmological Society, in 1910, and again in discussing Dr. Bryan's 
paper before the College of Physicians. The appearance and disappearance of these lid manifestations are obviously dependent upon relief from an obstruction to drainage in frontal, ethmoidal and antral empyema.

Congestion of the conjunctiva and lacrimation are very frequently present, the former extending at times to a definite conjunctival catarrh.

Emphasis must also be placed upon the presence of a fugitive episcleral congestion, resembling the episcleritis periodica fugax of Fuchs, or the Hutchinson "hot eye."

The dilated and tortuous episcleral vessels are deeper in hue than those of ordinary episcleritis, are said not to bleach under adrenalin, and are apt to be accompanied with very violent headache. These attacks may last for weeks, as in a case reported by de Schweinitz, before the cause is suspected.

To these may be added curious corneal phenomena mentioned by the same writer, namely, edema of the corneal epithelium, resembling that induced by cocain or a socalled wrinkling of the cornea. Posey and Gerber have seen herpetic cruptions associated with sinus disease.

Of the inflammatory ocular conditions, as is well known by every ophthalmic surgeon, none is at times more baffling than uveitis and iridncyclitis. One of the earliest reports associating this condition with sinus disease was by Dr. Posey in 1897, November 16, before the Ophthalmologic Section of the College, where the eyes of a laundry worker became blind and the ball shrunken from a violent uveitis, the nasal and sinus condition being due to inhalation of acid fumes. 'The involvement of the cornea, iris, ciliary body and choroid, constituting this well known uveal picture, may have a very varied etiology, such, for cxample. as syphilis, tuberculosis, entergenous intoxication of intestinal origin, gonorrhea and the toxic products from a diseased tooth, mouth, tonsil, skin, uterine cavity or accessory sinus, and it is perfectly possible that two of these sources of infection may be present at the same time, but since Ziem, Ebersbush (quoted by Brawley), and many other observers have associated these conditions with sinus disease, no study is complete that lacks sinus consideration. 
Disease and abscess formation in the region of the lacrimal sac, giving the symptoms strongly suggestive of lacrimal mucocele. may be the socalled prelacrimal abscess and have their origin in the lacrimoctlimoidal cells.

Alterations in refraction, the result of pressure from a dilated sinus is not an infrequently recorded observation and ciliary spasm and congestion, the outcome of nervous and lymphatic influences, may likewise contribute to the production of this phenomenon. Stewart of Portland (Ophthalmic Record, 1910) has scen three-fourths of a diopter against the rule disappear, after surgical treatment of a sinus.

I am entirely in accord with the view that these, and many others of the associated ocular and nasal conditions of indeterminate origin, but with at least a relaxed turgescence or socalled vasomotor hypertrophy may and should be relieved by general upbuilding, with iron for example, as suggested by MacWhinnie, or other tonics and supplemented by exercise, a change of climate, intestinal antiseptics and laxatives with appropriate local nasal treatment, with which you are all familiar, before operation is undertaken.

INFLAM MATORY ORTITAI, SYMPTOMS.

Inflammatory conditions of the orbit are present in and the result of nasal sinus disease in 60 per cent of the cases according to Birch-Hirschfeld. of I,eipsic, and indeed other authoritics assion an even more important role to them in the induction of orbital cellulitis: Posey, for example, maintains that fully nine-tenths of all cases of cellulitis are secondary to sinus affections, and St. Clair Thompson (I.ondon Ophthalmoscope, 1908, "The Frequency of Orbital Manifestation of Nasal Sinusitis"), belicves we should cease to regard orbital cellulitis as primary and should look to the nose and accessory sinuses for the infection. The inflammation often persists, notwithstanding the establishment of drainage by reason of the orbit being a closed cavity. The acute or chronic purulent sinuitis was due in Hirschfeld's cases to rhinitis, influenza, pneumonia, scarlet fever, diphtheria or traumatism.

The more frequent route of orbital infection from accessory sinus inflammation is by way of the floor of the frontal, the os planum of the ethmoid and the roof in the maxillary 
sinus, the symptoms varying as the condition is acute or chronic. In the acute variety the eyeball is tender, there is usually pain upon rotation, a socalled orbital neuralgia especially upon concentrating the gaze, and tumefaction at the upper inner or inner angle involving the inner third or half of the eyelids.

If the fundus can be seen the veins will be full, the edges of the disc veiled, the surface of the nerve too red, or edematous with confusion of sight, diplopia, edema of lids, conjunctival chemosis, periorbital pain increased upon pressure, dimness of vision and a varying proptosis of eyeball depending somewhat upon the primary source of the swelling, being forward, downward and outward in front sinuitis, with limitation of upward and inward movement, more nearly directly forward in sphenoid disease, and forward, downward and inward (Skillern) in ethmoiditis.

The completion of the symptom complex will be dependent upon the degree of cellulitis and its extension into an orbital abscess.

The rapidity with which an orbital cellulitis secondary to an acute ethmoiditis may proceed to a fatal issue is illustrated in the case of Clegg, in which a girl of nineteen previously perfectly well, developed sudden headache and vomiting with great edema of the lids and reduction of vision to light perception. Temperature and pulse were normal. Drainage of orbit brought temporary relief, but death ensued in four days.

Hilfrich, K. J., in a paper upon "Intraorbital Complications in Acute and Chronic Accessory Sinus Disease," Zeitschrift fur Ohrenkrankheiten, page 31, emphasizes the fact that swelling of the contents of the orbit causing protrusion, limitation of movement of the eyeball, diplopia and headache, marking the acute onset of exophthalmos, may be at times the only external sign of accessory sinus disease.

Mucoceles from the frontal and ethmoid are apt to be marked by the presence of a quiet tumor at the upper and inner or inner aspect of the orbit, dependent upon the involved sinus without active inflammatory symptoms causing displacement of the orbital contents with resulting exophthalmos down and out. This may be combined with polypoid formation and evident ethmoid disease or as in a case of Reber's 
can exist with an entirely negative rhinogenous finding. In the latter case a spray of antipyrin, cocain and adrenalin several times a day for about ten days suddenly resulted in a gush of thick jellylike fluid from her throat, causing a complete disappearance of all the symptoms.

These mucuceles may result in a periostitis of the frontal floor or over the os planum. A subperiosteal abscess may result and may either be encapsulated or penetrate the skin of the lid.

Axenfeld maintains that the distinction between osteoma and mucoceles, many of the symptoms of which are allied, nay be made by the $\mathrm{X}$-ray.

The enlargement caused by mucoceles is often of almost bony hardness. suggesting an exostosis. A tense parchment like appearance is apt to be followed by crepitation or fluctuation.

The siow growth permits an ocular adjustment, therefore there is frequently no diplopia and the failure of the optic nerve to show any radical departure from normal is explained by its straightening at the sigmoid flexure without pronounced stretching (Posey).

Gummata of the upper inner angle of the orbit may present symptoms closely similating mucocele distension as in a case of Zentmayer's and a second of F. C. Parker, and here when doubt exists as to each diagnosis the modem syphilis tests are invaluable.

The ocular changes secondary to sinus disease appear usually in adult life, but a number of well recorded cases appear in infancy and early childhood.

Drs. Frederick Krauss and William Campbell Pusey of this city, for example, have reported cases of orbital abscess secondary to maxillary sinuitis. In Dr. Krauss' case the disease appeared at four months and in Posey's between one and three years. Posey had originally regarded them as osteomalacial in origin.

Onodi records the size of the maxillary sinus at one year of age as 5 by 3 millimeters up to 19 by 8 millimeters in size, and in fetus of six and one-half months the same author found a maxillary sinus 3 by 1.5 millimeters. 


\section{OCULAR MUSCLF: INVOLVEMENT.}

This aspect of the subject has been carefully elaborated by Savineau, Peyser, Galezowski, Rernheim, Posey, Reber and others.

Interference with the mobility of the eye usually results from mechanical displacement associated with a distended sinus wall or from the presence of exudation, yet asthenopia, paretic or paralytic conditions of the ocular muscles do occur in sinuitis entirely apart from such displacements and occasionally may be an accompaniment of even a mild sinus involvement. These later cases, as Bernheim and Skillern suggest, are apt to be the result of the selective action of a toxin just as is true of the infectious discases, autointoxication and influenza.

The anatomic relations will readily point to the frontal cells as responsible for involvement of the superior rectus, superior oblique and the levator, and Savineau has associated the internus and accommodative muscle involvement with frontal disease, although the lack of power in the interni usually points to ethmoidal diseases and that of the inferior rectus and inferior oblique are most apt to indicate antral disturbance.

The intimate association of the nerves which supply the ocular muscles with the outer wall of the sphenoid will readily explain in part the muscle disturbance, and disease of the sphenoid is regarded by some authorities as the most frequent cause of muscle involvement of sinus origin. The ethmoid frontal and antrum probably offend in the order mentioned.

In acute cases pareses or paralyses are occasioned by direct inflammatory infiltration of the long flat belly of the muscles themselves as they lie close to the sinus walls or by involvement of the nerves as they enter the orbit.

In many instances diplopia is not complained of, but is detected by the red glass and a search in the peripheral field. Diagnostic help is gained by noting the pain when the eye is turned in the position that calls forth the greatest movement of the affected muscle.

The use of the eyes is difficult and painful, confusion of vision, vertigo and reflex gastric disturbance are frequently present. 
Alternate dilatation and contraction of the pupil is reported by McBean of Chicago in a paper upon variations in the sphenoid sinus (ANxills OF OTOLOCY, LARYNGOLOGY .AND RHINOLO(; , 1914) and two cases of pupillary dilatation in sphenoid disease are reported by Sluder.

The condition was one of acute sphenoiditis in McBean's case, with pain back of eye and in occiput with severe asthenopia. In a few days wide dilatation of pupil was followed by myosis, with spasm of accommodation. All local medication was resisted and the case cured by an autogenous vaccine. Mclean regards the explanation of the phenomena as an irritation of the carotid plexus of the sympathetic producing mydriasis, and considers that later motor oculi irritation produced myosis.

Baumgarten and Lapersonne are quoted by Onodi as also having seen oculmotor paresis and paralysis from sphenoidal disease.

The picture of complete palsy of the levator palpebrarum (right) frontal sinus empyema is apt to include severe neuralgic pain in the supraorbital and frontal region with marked tenderness upon tapping. In one case reported by Posey the globe movements were unrestricted. Conservative treatment to the frontal sinus by Dr. George 13 . Wood in this instance caused a rapid improvement. 'The' close apposition of the muscle to the sinus floor and the absence of sphenoid and ethmoid disease established a diagnosis of direct involvement.

It is not always easy to differentiate between edema and paralytic ptosis, each at times being fugacious and recurrent.

In the more chronic types of sinuitis the disturbance of the eyeball is slow because the sinus distension is very gradual and the muscles adjust themselves to varying conditions.

The socalled rheumatic ocular palsies are thought by a number of atthorities to be more often due to sinus disease.

It is perfectly well known that many of these ocular palsies are due to specific disease and every oculist of experience has seen cures result from large doses of the iodids, but if the possibility of sinus origin be kept in mind we will frequently produce a rapid cure by the sinus route. 
OCLLAR FUNDES CHANGTS UNACCOMPANIFII HY HATIRNAL INFLAMM.ITION.

An exhaustive ophthalmoscopic and perimetric examination becomes of especial importance in those cases of sinuitis in which changes in the funclus oculi are present without any external signs of orbital or ocular inflammation. These changes may be retrobulbar neuritis-a papilloedema or choked disc, an optic neuritis-neuroretinitis, thrombosis or phlebitis (de Schweinitz), retinal detachment and finally definite blind or blurred areas in the visual field without ophthalmoscopic evidence of ocular disease.

The areas of greatest significance in the study of intraocular disturbances are two in number, first the macula with its acute recognition of white and color, and second the optic nerve at its point of entrance into the eyeball, the latter being the socalled "silent" area or the blind spot of Marriot.

The presence of symptoms of optic nerve involvement (as emphasized strongly by distinguished authorities) are the most dangerous of the sinus pointings and if not relieved by suitable drainage may be followed by atrophy. If these changes are unilateral they especially call for sinus study.

The importance of repeated field examination in arriving at a more definite diagnosis is emphasized by such a case as reported by Heed, where a female of thirty-nine complained of blurred vision and vertigo and in which a fundus examination showed only a suspicious hyperemia of the disc but with normal fields. Five days later an active neuritis with contracted fields existed. Treatment to ethmoid cells caused a rapid cure.

The presence of a central scotoma in the absence of any demonstrable ocular involvement in the media, retina or choroid points definitely to a macular lesion arid is pathognomonic of an axial orbital or retrobulbar neuritis, which is ordinarily caused by the circulation of an infecting agent in the blood, this toxemia inducing an interstitial inflammation of the socalled papillomacular bundle of the nerve most marked in the optic canal. For these fibers certain toxic products have a special affinity, and the scotoma when present is usually first for colors and later for white. 
This toxemia as a rule results in a temporal pallor of the disc, but the discovery of the scotoma precedent to the change in the appearance of the disc usually points to the need of immediate improvement of sinus drainage and hence may. warrant operative interference when no demonstrable signs of sinus disease are present. If the infecting agent manifests its baneful influence on the intraocular end of the nerve as a choking of the disc, and actual neuritis or further extension into the retina, these significant ophthalmoscopic pictures can readily be determined.

The perimetric findings associated with or the direct outcome of sinus inflammation, have been carefully studied by a considerable group of observers, prominent among whom are: Birch-I Iirschfeld, Fuchs, Jessup. Zien, Van der Hoeve, dde Kleyn, Markbreiter, Ramsey, Southerland, Samuel I). Risley, de Schweinitz, Knapp, Reber, J. Norman Risley, MacWhinnie, F. J. Parker, Friedenberg and Peter.

There may be concentric or irregular contractions both for form and colors and occasionally bitemporal hemianopsia (E.vans-Pirmingham), but the scotomas of varying types are more generally present, have more diagnostic significance and indeed those of the socalled peripapillary variety have been regarded by some observers as practically pathognomonic. As the sinuitis is nore usually unilateral, so is the central scotoma. This fact has received special enphasis by Wecks of New York, but this will of course vary with the extent of the sinus involvement and may be bilateral and be present both for form and colors. The scotomata of the more usual toxic varieties caused by the various systemic toxemias are much more apt to be bilateral.

In our study of central scotoma three important groups representing varying types of causation must be considered from a differential viewpoint.

First: Toxic amblyopias caused by alcohol, tobacco, lead, arsenic, iodoform, stramonium, carbonioxịde, mercury, opium, malefern, etc.

Second: Intestinal toxemia, syphilis, tuberculosis, diabetes, gout (socalled), gonorrhea, influenza, malaria, the infectious fevers.

Third: The mouth, teeth, tonsils and accessory nasal sin- 
uses, and it has been generally belicved that the posterior sinuses are the special offenders.

It must always be kept in mind that one or more of these systemic conditions may be responsible for a toxemia which induces the nerve changes or may result in the sinus empyema, which in turn may be the direct excitant of the nerve or retinal lesion. Hillizter (W'ien. klin. Rundschon, October 8, 1911) believes optic nerve conditions due to involvement of the ethmoid and sphenoid sinuses are as a rule diagnosticated by exclusion.

In the absence of intoxications with a negative Wassermann the presence of an axial or retrobulbar neuritis, acute or chronic (especially if unilateral, since in the former condition the nerve disturbance is usually bilateral), the possibility of a disseminated sclerosis should be carefully considered and under such conditions disturbances of gait-sensation, reflexes should be studied, and indeed if a sinus infection is present, such as ethmoiditis, for example, combined with the symptoms of disseminated sclerosis, as was true in a case reported by Shumway, the possibility of the toxin from the purulent focus causing the nerve degeneration as suggested by that author must likewise be kept in mind.

The differentiation betwcen the fields of vision and intraocular states due to sinus disease and those due to or associated with general toxemias, in which no sinus disease was suspected nor present, are, as pointed out by S. D. Risley, sometimes exceedingly difficult to make.

Central scotoma, narrowing of ficlds, edema of fundus, veiling all details, large dark tortuous veins, hemorrhages in fibre layer of retina or at the macula may be present and yet it may be impossible to demonstrate sinus disease. On the other hand, all of above symptoms may be present in association with disease of one of the sinus in the anterior segment of the skull and it is not always easy to say they were caused by it, as both. the sinus and ocular disease were associated with and probably caused by the same systemic toxemia.

Paracentral scotomas of horseshoe shape, disappearing in segments, are described by MacWhinnic and a similar variety is emphasized by de Schweinitz, the paracentral often be- 
coming hemianopic and resembling the hemianopic scotoma of 'Treitel.

The crescentic field defects, such as reported by the same author in a case of posterior ethmoiditis combined with tooth abscess, may be part of a ring scotoma, either previously present or not yet completely formed and easily influenced by measures to remove the toxemia, that is to say, treatment of sinus and tooth.

'The ring scotomatas may become central and usually improve and disappear with adequate drainage unless permanent damage has resulted from the pressure or toxemia.

The presence of a ring scotoma according to MacWhinnie (quoted by Friedenberg), would indicate accessory sinus involvement even when the retained infection was not evident to the probe. When inspection of the nose points to definite sinus involvement, especially posterior, a typical field will be an enlargement of the blind spot and a paracentral scotoma.

The nerve lesions in central amblyopia consist in edema and proliferation of the glia cells and later possible destruction of the nerve fibre (Birch-Hirschfeld). The cause, according to Birch-Hirschfeld, is venous stasis and toxic agencies.

Bryan believes that nerve toxemias can be caused by mucoccles as well as by abscesses and the symptoms often extremely difficult to explain may be the result of anomalies in the posterior cavities. The sphenoid may be in relation to both nerves. The posterior ethmoid may be, on the other hand, in close proximity to one and not to the other, and furthermore, it is Bryan's opinion-that the presence of bilateral nerve involvement may be explained by transference by way of the chiasm without necessarily indicating the presence of a bilateral sinuitis.

In such a study as this special emphasis should be laid upon the work of Van der Hoeve and its further development and confirmation by de Kleyn.

The enlargement of the blind spot constituting the socalled peripapillary or Van der Hoeve scotoma, has been thought to be pathognomonic of involvement of the posterior group of cells (sphenoid and posterior ethmoid); this toxemia ex- 
pressing itself upon the peripapillary bundle as the first portion involved in a retrobulbar neuritis.

In attempting to outline a Van der Hoeve phenomenon it must be remembered that the optic nerve as it enters the eye has no ganglion cells, no rods nor cones, and is therefore blind.

This blind spot is located $15^{\circ}$ to the temporal side of the actual point of fixation. In hyperopic eyes it is further away as far as $19^{\circ}$, while in myopic eyes it is down to $11^{\circ}$. As to the important question, what actually constitutes an enlargement of the blind spot, Van der Hoeve considered over $6^{\circ}$ in the horizontal diameter as suspicious and over $7^{\circ}$ as too large.

Van der Hoeve found the peripapillary scotoma only in posterior sinus disease, and maintains that central scotoma, always secondary to sinus disease, appeared later if at all: whereas in toxic retrobulbar neuritis this symptom and the Van der Hoeve scotoma were present simultaneously. De Kleyn noted that the enlargement of the blind spot for colors preceded that for a white.

The presence of this symptom according to some authorities justifies an operation on the affected sinus if no other cause for the blind spot enlargement can be found.

These deductions we will probably modify by reason of the more recent work of Markbreiter in an important paper on the changes in the visual ficlds in diseases of the nose and accessory sinuses, in which he reported results of investigations of the fields in one hundred cases of empyema, carefully excluding all cases in which the eye itself manifested any changes that might cause any visual field disturbance.

In this series of cases sixty-three involved the anterior group of cells, and of this series seventeen were frontal and thirty-one maxillary, three anterior ethmoid and frontal, nine involved the posterior group, three were cases of pansinuitis, and in the remaining twenty-five the exact location is not specified.

Visual field changes were found in seventy of the hundred cases, although the fundus in each case was normal and the vision perfect. This, it seems to me, is a very important observation. 
In the anterior empyemas, field changes were present in forty-eight out of sixty-three cases and in seven out of nine in the posterior group.

Of the seventy cases of defective fields the blind spot enlargement was present in fifty-two, seven showed central and eleven other forms of field defect, such as island ring defects and peripheral contraction.

Markbreiter has never seen enlargement of blind spot pass into central scotoma.

In eleven out of thirty-seven nonsuppurative diseases of the sinuses, field changes were present.

The further important deduction from this study is, that while enlargement of the plind spot constitutes the most frequent of the nerve manifestation of accessory sinus disease, it presents no diagnostic features by which we can positively say whether the anterior or posterior group of cells is involved.

The fact, however, that enlargement of the blind spot may sometimes be present, either with or without surrounding rings of color scotoma, and the further fact that these scotomas may antedate by some considerable time organic changes in the nerve head or other gross ophthalmoscopic alteration, is an exceedingly important observation of Van der Hoeve, de Kleyn, Onodi, and has been confirmed by a number of American observers (de Schweinitz, Norman Risley), and while it may not definitely indicate which sinus or group of sinuses is involved, it constitutes a very significant signpost in the direction of sinus disease.

Optic neuritis and neuroretinitis may be caused by an inflammation of any of the nasal sinuses. In a case reported by Arnold Knapp resection of the anterior half of the middle turbinal and curetting of the posterior ethmoid caused a return to normal vision and fundus, and eliminated a central relative scotoma for white and colors.

An optic neuritis in which the summit of the disc was plus four with an ocular proptosis and reduction of vision to one-fifth of normal, in which frontal ethmoidal drainage by incision under the orbital rim from a point under the middle of the front inward to the nasal front and downward along 
its border, with complete recovery, was reported by Dr. Risley. This procedure was adopted in 1903, and the patient, whom I saw this past week, has scarcely any deformity and normal vision. This author much prefers the procedure here adopted as offering adequate drainage and a very inconsiderable scar.

Monolateral optic neuritis may exist for a prolonged period with normal vision as in the case reported by Rau, where a systemic treatment caused marked improvement in a case lasting a year. Recurrence was noted in two years with decreased vision, frontal headache and pain on percussion over the frontal sinus. Examination now showed a maxillary sinuitis of the same side, operation upon which cured the neuritis and largely restored vision.

This case emphasizes the fact that pain is not an absolutely reliable guide, as none was present over the maxillary and all over the frontal.

The tolerance of the optic nerve and retina to interference of toxic products of or direct pressure from ethmoidal empyema, is well illustrated by a case reported by Dr. S. D. Risley ten years ago, before the association of these conditions was by any means so generally recognized. It likewise confirms an equally important observation that the ordinaty depleting, absorbing and eliminating measures so generally applied to cases of neuroretinitis with flame-shaped hemorrhages and contraction of the field, will frequently cause amelioration of all the symptoms. The recrudescence of the ocular condition in this instance caused the consulting neurologist to fear intracranial involvement. Operation on the anterior ethmoid cells and consequent drainage of frontal and ethmoid resulted in a complete cure, the latter not having been performed for two years after the initial symptoms.

I venture the opinion that all of us today would immediately take the sinuses into account at an initial visit in the presence of a unilateral neuroretinitis, with or without hemorrhages.

The case of a physician recorded by Parker of New York with concentric contraction, central and Van der Hoeve scotomas, varying with the conditions of the sphenoid and posterior ethmoid cells combined with double papilloedema. is of special interest. 
The maxillary antrum was also involved, but the cure of the ocular condition followed operative treatment directed to the ethmoid and sphenoid sinuses, vision at one period being reduced to shadows in each eye and returning to normal only when the last posterior infected cell had been drained.

Retinal thrombosis as symptomatic of sinus disease is referred to by a number of writers, Wendell Reber reporting a case of thrombotic involvement of the central retinal vein which was negative to all types of examination, even rhinologic. In his characteristic phraseology he pictures the nasal disease as sweeping in, doing its damage and possibly disappearing by the time the retinal disease is apparent, notwithstanding a negative examination so far as empyema was concerned he insisted upon rhinological local nonoperative treatment and a cure resulted in four weeks.

Reber believes many of these cases of thrombosis of the central vein are due to undiagnosed latent obscure sinus disease which need not be purulent.

In this perhaps too prolonged recital many vital points have been left for consideration and review by the specialists present whose extensive experience and reputation entitle their pronouncments to the most distinguished consideration.

These subjects include the importance of blood study with hemoglobin determination and differential count, the relation of blood pressure, intestinal toxemia and the varying infections, the conservation of tissue as opposed to its destruction and removal, the value and limitation of vaccine therapy, the bacterial study as indicating the extent to which conservative treatment is justified, the limitations of X-ray and transillumination as adjuncts to accurate diagnostic study, the best conservative nasal treatment, which, according to one author (MacWhinnie), will cure ninety-seven per cent of these cases, the value of suction in the conservative management, and finally the most approved operative intervention so often essential to the preservation of vision and at times to the prevention of meningitic involvement.

The important opinions of the participants in the discussion of the evening have been dug out of the mines of the years of toiling in an effort (in the apt phraseology of Edward Jack- 
son) to "Push back the ever widening margin of the unknown."

If our interdependence in our closely allied fields of endeavor has been given an emphasis, the purpose of the writer will have been achieved. 